\title{
MANUSEIO DA SÍNDROME COMPARTIMENTAL ABDOMINAL EM UNIDADE DE TRATAMENTO INTENSIVO
}

\section{INTENSIVE CARE MANAGEMENT OF THE ABDOMINAL COMPARTMENT SYNDROME}

\author{
Luiz Carlos Von Bahten,TCBC-PR ${ }^{1}$; Paulo de Souza Fonseca Guimarães ${ }^{2}$
}

\begin{abstract}
RESUMO: A síndrome compartimental abdominal (SCA) decorre de um aumento agudo na pressão intra-abdominal (PIA), promovendo alterações fisiológicas adversas devido ao acometimento dos principais sistemas orgânicos, podendo levar a falência orgânica e óbito. Objetivo: Avaliar a SCA em pacientes com hipertensão intra-abdominal (HIA) admitidos na unidade de terapia intensiva (UTI). Método: Foi realizado um estudo de 548 pacientes submetidos a laparotomia, necessitando de UTI, durante o período de janeiro de 1997 a março de 2001. Resultados: A SCA foi identificada em 29 pacientes (5,29\%). Analisando-se o valor máximo de PIA, $9(31,03 \%)$ foram grau II, $10(34,48 \%)$ foram grau III e $10(34,48 \%)$ foram grau IV. Dezoito $(62,07 \%)$ foram reoperados e o fechamento temporário foi realizado em $6(20,69 \%)$. A mortalidade global foi de $68,97 \%$, sendo que a SCA grau II teve 55,56\% de mortalidade; a de grau III, $50 \%$; a de grau IV, $100 \%$ e a mortalidade dos pacientes reoperados, $61,11 \%$. Conclusão: A SCA acarreta elevada mortalidade, mesmo com a reoperação precoce e adequado manejo em UTI, devendo-se manter maiores cuidados na sua identificação e prevenção (Rev. Col. Bras. Cir. 2006; 33(3): 146-150).
\end{abstract}

Descritores: Pressão; Abdome; Hipertensão; Síndrome de compartimento; Síndrome de compartimento/complicações.

\section{INTRODUÇÃO}

A síndrome compartimental abdominal (SCA) é conseqüente de um aumento agudo na pressão intra-abdominal (PIA), o que promove alterações fisiológicas adversas, devido ao acometimento principalmente dos sistemas cardiovascular, renal e pulmonar, o que pode levar a falência orgânica e óbito. É freqüentemente observada em pacientes traumatizados portadores de coagulopatia, sendo as alterações adversas prontamente revertidas pela descompressão abdominal ${ }^{1,2,3,4}$.

O objetivo deste trabalho é avaliar a SCA em pacientes com hipertensão intra-abdominal (HIA) admitidos na Unidade de Terapia Intensiva (UTI) do Hospital Universitário Cajuru, um centro de referência de emergências cirúrgicas da cidade de Curitiba.

\section{MÉTODO}

Estudo retrospectivo realizado durante o período de janeiro de 1997 a março de 2001, no qual foram analisados 548 casos de pacientes submetidos a laparotomia e que necessitaram de UTI.

Os casos nos quais ocorreu a SCA foram analisados quanto ao fator etiológico, às lesões orgânicas encontradas, aos procedimentos cirúrgicos realizados e ao manejo na UTI. Avaliou-se o tempo de permanência na UTI, valores de PIA mínimo e máximo, complicações, utilização de relaxantes musculares, necessidade de reoperação e mortalidade.

Na UTI a PIA foi mensurada indiretamente através de coluna d'água, através da pressão intravesical e a SCA foi graduada de acordo com os valores encontrados, conforme o sistema de graduação da Associação Americana de Cirurgia do Trauma, a qual define SCA grau I (PIA entre 10 a $15 \mathrm{~cm}$ $\mathrm{H}_{2} \mathrm{O}$ ), grau II (PIA entre 15 e $25 \mathrm{~cm} \mathrm{H}_{2} \mathrm{O}$ ), grau III (PIA entre 25 e $35 \mathrm{~cm} \mathrm{H}_{2} \mathrm{O}$ ) e grau IV (PIA maior que $35 \mathrm{~cm} \mathrm{H}_{2} \mathrm{O}$ ) ${ }^{2,3,4}$.

\section{RESULTADOS}

Dos 548 pacientes submetidos a laparotomia e que necessitaram de UTI, a SCA foi identificada em 29 (5,29\%). A faixa etária dos pacientes variou de três a 59 anos, com uma média de 28,96 anos, sendo $21(72,41 \%)$ homens e oito $(27,59 \%)$ mulheres. O tempo de permanência na UTI teve uma variação de um a 76 dias (média de 17,77 dias). Nos sobreviventes o tempo de permanência na UTI variou de um a 26 dias (média de 10,5 dias) e o tempo de permanência hospitalar variou de 10 a 76 dias (38,6 dias).

A SCA teve etiologia traumática em 26 pacientes $(89,66 \%)$, sendo o trauma abdominal penetrante presente em $14(48,28 \%)$, dos quais o ferimento por arma de fogo ocorreu em $9(31,05 \%)$ e o ferimento por arma branca, em cinco $(17,25 \%)$. O trauma abdominal contuso ocorreu em 12 (41,38\%), dos quais cinco $(17,25 \%)$ foram por colisão automobilística, qua-

\footnotetext{
1. Médico Cirurgião Geral do HUC - PUC - PR; Professor Adjunto da Disciplina de Anatomia Médica da PUC - PR; Professor Assistente da Disciplina de Trauma da UFPR; Mestre em Cirurgia Geral pela PUC - PR; Doutorando do Curso de Pós- Graduação em Cirurgia do Trauma UNICAMP.

2. Médico Residente do Serviço de Cirurgia Torácica e Endoscopia Respiratória da Aliança Saúde (Hospital Universitário Cajuru - Santa Casa de Misericórdia).
} 
tro $(13,8 \%)$ por atropelamento, dois $(6,9 \%)$ por queda de nível e um $(3,45 \%)$ por agressão. Somente três casos $(10,35 \%)$ não foram de origem traumática, com a SCA desenvolvida a partir de sepse consequiente a abdome agudo infeccioso de corrente de apendicite aguda perfurada, de colecistite complicada e de pancreatite necro-hemorrágica.

Dos pacientes traumatizados, $16(55,17 \%)$ foram tranportados pelo SIATE (Serviço de Atendimento Integrado ao Trauma), tendo o tempo de chegada ao hospital até o centro cirúrgico variado de 15 minutos a oito horas, com média de uma hora e 57 minutos. O tempo de cirurgia variou de uma hora e 30 minutos a cinco horas, com uma média de três horas e dez minutos.

Foram encontradas 59 lesões intra-abdominais durante a cirurgia inicial, sendo o fígado acometido em 12 (20,34\%), o intestino delgado em 12 (20,34\%), pâncreas em oito $(13,56 \%)$, intestino grosso em sete $(11,86 \%)$, grandes vasos em sete $(11,86 \%)$, baço em cinco $(8,48 \%)$, estômago em três $(5,09 \%)$, rins em três $(5,09 \%)$ e mesentério em dois $(3,39 \%)$. Destaca-se que $83,33 \%$ das lesões de intestino delgado foram duodenais, identificadas em 10 pacientes $(34,48 \%)$, ocorrendo lesão do complexo duodeno-pancreático em quatro $(13,79 \%)$. Ocorreram 16 lesões extra-abdominais, presentes em sete $(24,14 \%)$ pacientes, das quais lesões contusas ocorreram em seis $(20,69 \%)$ sendo fraturas em membros inferiores presentes em cinco (17,24\%), trauma crânio-encefálico em três $(10,35 \%)$, trauma torácico em dois $(6,9 \%)$, fratura pélvica em um $(3,45 \%)$ e fratura em membros superiores em dois (6,9\%). As lesões extra-abdominais decorrentes de trauma penetrante ocorreram em apenas um paciente vítima de ferimento por arma de fogo, o qual apresentava três lesões, sendo uma no tórax, a as outras nos membros superior e inferior direitos.

Dos procedimentos cirúrgicos que foram realizados, a enterorrafia foi realizada em $11(17,16 \%)$, hepatorrafia em nove $(14,04 \%)$, pancreatorrafia em cinco $(7,8 \%)$, esplenectomia em cinco $(7,8 \%)$, cavorrafia em quatro $(6,24 \%)$, drenagem torácica em quatro $(6,24 \%)$, colorrafia em três $(4,68 \%)$, colecistectomia em três $(4,68 \%)$, gastrorrafia em três $(4,68 \%)$, toracotomia com clampeamento aórtico em dois $(3,12 \%)$, enterectomia em dois $(3,12 \%)$, portorrafia emdois $(3,12 \%)$, pancreatectomia distal em um $(1,56 \%)$, colectomia parcial em um $(1,56 \%)$, nefrectomia em um $(1,56 \%)$, nefrorrafia em um $(1,56 \%)$, mesenteriorrafia em um $(1,56 \%)$, aortorrafia em um $(1,56 \%)$, retorrafia em um $(1,56 \%)$, derivação bileo-digestiva em um( $1,56 \%)$ e apendicectomia em um $(1,85 \%)$.

Após a cirurgia inicial os pacientes foram transferidos para a UTI, onde a PIA foi mensurada através de sonda vesical a qual foi mantida por um período médio de 3,54 dias. No momento inicial de mensuração da PIA, 10 pacientes $(34,48 \%)$ não apresentavam SCA, sete $(24,14 \%)$ eram grau I, oito $(27,58 \%)$ eram grau II e quatro $(13,79 \%)$ eram grau III. Durante o momento de maior valor da PIA, nove $(31,03 \%)$ eram grau II, $10(34,48 \%)$ eram grau III e $10(34,48 \%)$ eram grau IV (Figura 1). A reoperação ocorreu em 18 (62,07\%), dos quais três $(10,35 \%)$ eram SCA grau II, oito $(27,59 \%)$ eram grau III e sete $(24,14 \%)$ eram grau IV. Dos pacientes reoperados, $12(41,38 \%)$ foram a óbito e apenas seis $(20,69 \%)$ sobreviveram, dos quais um $(3,45 \%)$ era grau II e cinco $(17,24 \%)$ era grau III.

De acordo com a Tabela 1, o fechamento temporário foi realizado em seis $(20,69 \%)$, sendo a bolsa de Bogotá (Figura 2) utilizada em cinco $(17,24 \%)$ e o fechamento com pontos totais separados em apenas um $(3,45 \%)$.

Relaxante muscular do tipo brometo de pancurônio (Pavulon $囚$ ) foi utilizado em 18 pacientes $(62,07 \%)$, dos quais cinco $(17,24 \%)$ eram grau II, sete $(24,14 \%)$ eram grau III e seis $(20,69 \%)$ eram grau IV. Destes pacientes, sete $(24,14 \%)$ sobreviveram, sendo dois $(6,9 \%)$ grau II e cinco $(17,24 \%)$ grau III. Esta droga foi administrada na dose de $0,1 \mathrm{mg} / \mathrm{kg}$ endovenosa, com monitorização eletromiográfica da musculatura hipotenar ou músculo reto abdominal.

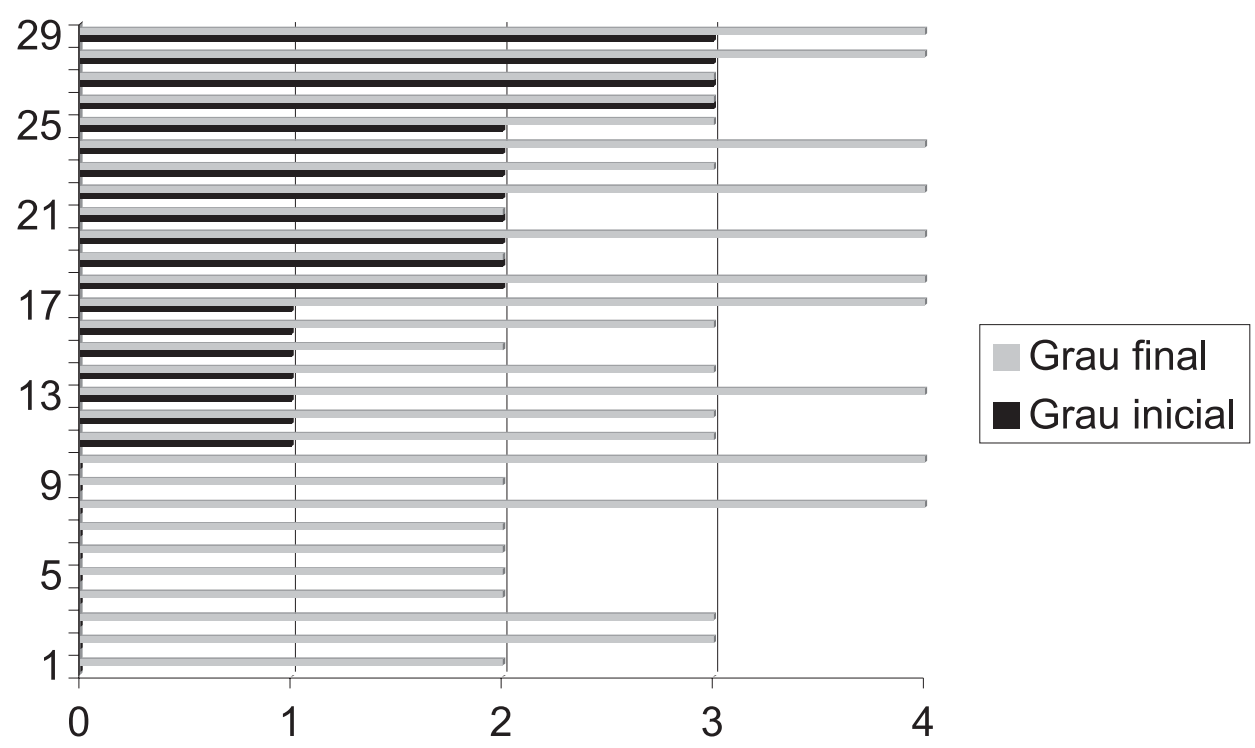

Figura 1 - Evolução da SCA por paciente*.

"Numa forma individualizada, no eixo das ordenadas em função do grau de SCA visto no eixo das coordenadas. 
Tabela 1 - Análise dos casos de fechamento abdominal temporário*.

\begin{tabular}{lcccccccccc}
\hline Caso & Idade & Sexo & Mecanismo & $\begin{array}{c}\text { Grau } \\
\text { Mínimo }\end{array}$ & $\begin{array}{c}\text { Grau } \\
\text { Máximo }\end{array}$ & $\begin{array}{c}\text { Dia } \\
\text { UTI }\end{array}$ & $\begin{array}{c}\text { Tempo } \\
\text { UTI }^{* *}\end{array}$ & $\begin{array}{c}\text { Tempo } \\
\text { Total }^{* * *}\end{array}$ & $\begin{array}{c}\text { Tipode } \\
\text { fechamento }\end{array}$ & Óbito \\
\hline 1 & 3 & F & Atropelamento & GI & GIII & 5 & 6 & 6 & Bolsa bogotá & Sim \\
2 & 43 & F & Atropelamento & GII & GII & - & 3 & 4 & Bolsa bogotá & Sim \\
3 & 8 & M & Agressão & GII & GIV & 4 & 7 & 20 & Bolsa bogotá & Não \\
4 & 27 & M & FAB & $<10$ & GII & 2 & 5 & 10 & Bolsa bogotá & Não \\
5 & 19 & M & FAF & GII & GIV & 2 & 3 & 3 & Pontos totais & Sim \\
6 & 16 & M & FAF & GII & GIV & 10 & 18 & 18 & Bolsa bogotá & Sim \\
\hline
\end{tabular}

*Todos os pacientes acima foram reoperados.

** Tempo de permanência em dias.

Caso 1. Lesões: fígado GIV, baço GV.

Caso 2. Lesões: TCE (fratura de base de crânio), fratura de pelve, hematoma retroperitonial.

Caso 3. Lesões: fígado GIV. * O gráfico mostra os pacientes, numa forma individualizada, no eixo das ordenadas em função do grau de SCA visto no eixo das coordenadas.

Caso 4. Lesões: intestino delgado, hematoma retroperitonial.

Caso 5. Lesões: face, membros, tórax (drenagem torácica), fígado GII.

Caso 6. Lesões: fígado GIII, artéria gástrica esquerda, artéria esplênica, veia porta.

Vinte pacientes morreram $(68,97 \%)$, sendo cinco $(17,24 \%)$ grau II, cinco $(17,24 \%)$ grau III e $10(34,48 \%)$ grau IV. Dos nove $(31,03 \%)$ que sobreviveram, quatro $(13,79 \%)$ eram grau II e cinco $(17,24 \%)$ grau III. As principais complicações encontradas foram coagulopatia em $10(34,48 \%)$, insuficiência renal em nove (31,03\%), sepse em seis $(20,69 \%)$, insuficiência respiratória em quatro $(13,79 \%)$, choque hipovolêmico em quatro $(13,79 \%)$, derrame pleural em quatro $(13,79 \%)$, hemorragia digestiva alta em três $(10,34 \%)$, acidose metabólica em três $(10,34 \%)$, hematúria macroscópica em três $(10,34 \%)$, pancreatite em três $(10,34 \%)$, coma em um $(3,45 \%)$, evisceração em um $(3,45 \%)$ e falência de múltiplos órgãos e sistemas em um $(3,45 \%)$.

\section{DISCUSSÃo}

O surgimento da SCA está diretamente relacionado com fatores que elevam a PIA, o que pode ocorrer após qualquer cirurgia abdominal, principalmente se evoluir com hemorragia ou se estiver relacionada ao traumatismo abdominal grave $^{5,6}$. Sua incidência não é precisamente definida, mas pode ser observada em 2 a $25 \%$ dos casos de traumatismo abdominal grave manejados por técnicas de controle de dano. Neste

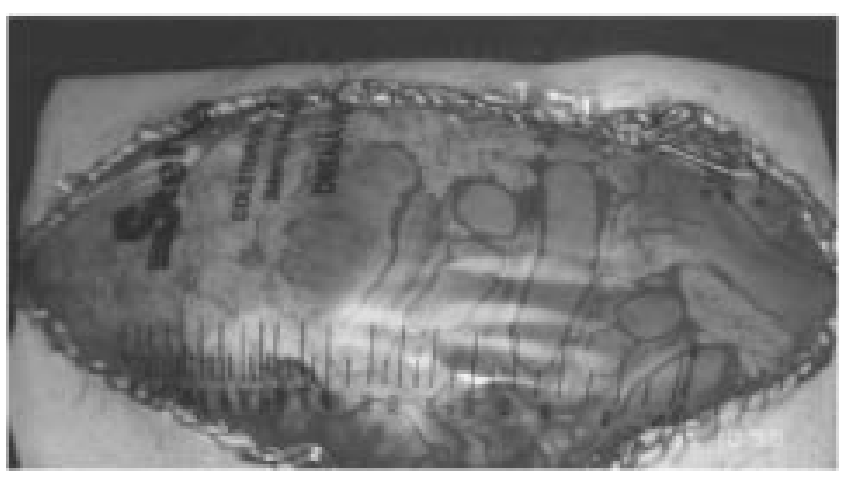

Figura 2 - Bolsa de Bogotá. trabalho sua incidência foi de 5,29\%, sendo a etiologia traumática predominante, observada em 26 pacientes $(89,66 \%)$, o que explica a faixa etária jovem (média de 28,96 anos) e o maior envolvimento de indivíduos do sexo masculino, observado em $21(72,41 \%)$. A gravidade do trauma abdominal pode ser notada pelo grande número de lesões intra-abdominais observado durante a laparotomia inicial (59 lesões), sendo o fígado e o intestino delgado os órgãos mais freqüentemente acometidos, observados em $12(20,34 \%)$.

O desenvolvimento da SCA promove alterações fisiológicas adversas em vários sistemas orgânicos:

- afeta o sistema cardiovascular, com recução do débito cardíaco, da força de contração e a complacência ventricular $^{2,3,7}$;

- compressão dos pulmões prejudicando a função respiratória; ocasionando deslocamento superior do diafragma. Oligúria progressiva, podendo chegar a anúria, o que pode ser explicado principalmente pela compressão da veia renal'; redução do fluxo sangüíneo esplâncnico, levando à isquemia intestinal, ulceração da mucosa gástrica, disfunção hepatopancreática e translocação bacteriana ${ }^{1,2,4,6,10}$; eleva a pressão intracraniana, pela obstrução funcional do retorno venoso cerebral via sistema venoso jugular ${ }^{11,12,13}$; afeta a perfusão da parede abdominal, principalmente se houver tensão durante o fechamento, causando deiscência com necrose e infecção da incisão cirúrgica ${ }^{4}$.

Analisando-se o valor máximo em que se atingiu a PIA mensurada durante a permanência na UTI, observou-se que $25(86,21 \%)$ sofreram acréscimo e apenas quatro $(13,79 \%)$ mantiveram seus valores de PIA estáveis (Figura 2). Dos pacientes que inicialmente não apresentavam SCA, seis $(20,69 \%)$ evoluíram para grau II, dois $(6,9 \%)$ para grau III e dois $(6,9 \%)$ para grau IV. Dos que inicialmente se apresentavam com SCA grau I, um $(3,45 \%)$ evoluiu para grau II, quatro $(13,79 \%)$ para grau III e dois $(6,9 \%)$ para grau IV. Dos que inicialmente se apresentavam com SCA grau II, dois $(6,9 \%)$ mantiveram-se em grau II, dois $(6,9 \%)$ evoluíram para grau III e quatro $(13,79 \%)$ 
evoluíram para grau IV. Dos que inicialmente se apresentavam com grau III, dois $(6,9 \%)$ evoluíram para grau IV. Na SCA grau I (PIA entre 10 a $15 \mathrm{~cm} \mathrm{H}_{2} \mathrm{O}$ ), apesar de poderem existir alterações fisiológicas significativas, a indicação de descompressão abdominal é duvidosa. No tratamento da SCA grau II (PIA entre 15 a $25 \mathrm{~cm} \mathrm{H}_{2} \mathrm{O}$ ) a necessidade do tratamento deve se basear na condição clínica do paciente, pois na ausência de oligúria, hipóxia ou pressões perigosamente elevadas nas vias respiratórias, é difícil justificar a descompressão abdominal, mas este grupo requer monitorização atenta. A maioria dos pacientes com grau III (PIA entre 25 a 35 $\mathrm{cm} \mathrm{H}_{2} \mathrm{O}$ ) necessita de descompressão, pois os sinais e sintomas de SCA podem instalar-se de forma súbita. Todos os pacientes de grau IV (PIA maior que $35 \mathrm{~cm} \mathrm{H}_{2} \mathrm{O}$ ) necessitam de descompressão pois podem evoluir para parada cardíaca em poucas horas ${ }^{7}$.

Neste estudo houve reoperação em 18 (62,07\%), dos quais três $(10,35 \%)$ eram grau II, oito $(27,59 \%)$ eram grau III e sete $(24,14 \%)$ eram grau IV. Apenas seis $(290,69 \%)$ dos reoperados sobreviveram, sendo um $(3,45 \%)$ grau II e cinco $(17,24 \%)$ grau III. A mortalidade da SCA grave é de $100 \%$ quando não revertida com descompressão abdominal e com a descompressão, a mortalidade varia de 42 a $68 \%$, principalmente devido a sepsis e efeitos tardios do choque hemorrágico ${ }^{6}$. Neste estudo a mortalidade da SCA foi de $68,97 \%$, sendo diretamente relacionada com o grau de SCA e nos pacientes reoperados a mortalidade foi de $66,67 \%$, sendo que as complicações mais freqüentes foram coagulopatias (34,48\%), insuficiência renal $(31,03 \%)$ e sepse $(20,69 \%)$.

Os pacientes que desenvolvem a SCA estão gravemente efermos e mesmo com os cuidados adequados de descompressão a mortalidade é elevada, sendo intimamente relacionada com as principais complicações da SCA, como a insuficiência renal, insuficiência respiratória, insuficiência hepática, necrose intestinal focal, necrose de ferida com deiscência, sepse e falência de múltiplos órgãos e siste$\operatorname{mas}^{14,15,16}$.

O prognóstico destes pacientes é afetado se houver atraso na descompressão abdominal dos pacientes com HIA, por isso medidas preventivas devem ser adotadas precocemente, como rapidez no encaminhamento destes pacientes ao centro cirúrgico, técnicas adequadas de controle de dano, manejo adequado na UTI com adequada mensuração de PIA, utilização de relaxantes musculares e rápida estabilização do paciente, prevenindo-se o desenvolvimento da cascata inflamatória da SCA, a qual pode ser irreversível, mesmo com medidas de suporte adequadas.

\begin{abstract}
Abdominal compartment syndrome (ACS) is attributed to acute increase in the intra-abdominal pressure (IAP), leading to adverse physiologic alterations because of involving the main organic systems and can lead to organic failure and obit. Background: the purpose of this work is to evaluate the intra-abdominal hypertension (IAH) and ACS conduct in patients submitted to intensive care unit (ICU). Methods: in this retrospective work were analysed 548 patients submitted to laparotomy and ICU, during the period from 1997, january to 2001, march. Results: ACS was noted in 29 patients (5.29\%). Analysing the maximum IAP value, 9 (31.03\%) were grade II, 10 (34.48\%) were grade III and 10 were grade IV. Eighteen (62.07\%) were submitted to reoperation and temporary closure was used in 6 (20.69\%). The global mortality was 68.97\%, the ACS grade II mortality was $55.56 \%$, the ACS grade III was 50\%, the ACS grade IV was 100\%. The reoperad patients mortality was $61.11 \%$. Conclusions: the ACS has a elevated mortality in spite of early reoperation and adequate management in ICU, and we need to identify this problem in a precocius way without delaying preventive actions.
\end{abstract}

Key words: Pressure; Abdomen; Hypertension; Compartment syndromes; Compartment syndromes/complications.

\section{REFERÊNCIAS}

1. Moore AF, Hargest R, Martin M, Delicata RJ. Intra-abdominal hypertension and the abdominal compartment syndrome. Br J Surg. 2004;91(9):1102-10.

2. Eddy V, Nunn C, Morris JA Jr. Abdominal compartment syndrome. The Nashville experience. Surg Clin North Am. 1997;77(4):801-12.

3. Sugrue M. Abdominal compartment syndrome. Curr Opin Crit Care. 2005;11(4):333-8.

4. Meldrum DR, Moore FA, Moore EE, Franciose RJ, Sauaia A, Burch JM. Prospective characterization and selective management of the abdominal compartment syndrome. Am J Surg. 1997;174(6):667-72; discussion 672-3.

5. Cheatham ML, Safcsak K. Intraabdominal pressure: a revised method for measurement. J Am Coll Surg. 1998;186(3):368-9. Corrected and republished from: J Am Coll Surg. 1998;186(5):594-5.
6. Balogh Z, Jones F, D'Amours S, Parr M, Sugrue M. Continuous intra-abdominal pressure measurement technique. Am J Surg. 2004;188(6):679-84.

7. Malbrain ML. Different techniques to mensure intra-abdominal pressure (IAP): time for a critical re-appraisal. Intensive Care Med. 2004;30(3):357-71. Epub 2004 Jan 17.

8. Ivatury RR, Diebel L, Porter JM, Simon RJ. Intra-abdominal hypertension and the abdominal compartment syndrome. Surg Clin North Am. 1997;77(4):783-800.

9. Hunter JD, Damani Z. Intra-abdominal hypertension and the abdominal compartment syndrome. Anaesthesia. 2004;59(9):899907.

10. Doty JM, Saggi BH, Blocher CR, Fakhry I, Gehr T, Sica D, Sugerman HJ. Effect of increased renal parenchymal pressure on renal function. J Trauma. 2000;48(5):874-7.

11. Walker J, Criddle LM. Pathophysiology and management of abdominal compartment syndrome. Am J Crit Care. 2003;12(4):367-71; quiz 372-3. 
12. Bloomfield GL, Ridings PC, Blocher CR, Marmarou A, Sugerman HJ. A proposed relationship between increased intra-abdominal, intrathoracic, and intracranial pressure. Crit Care Med. 1997;25(3):496-503.

13. McNelis J, Marini CP, Simmus HH. Abdominal compartment syndrome: clinical manifestations and predictive factors. Curr Opin Crit Care. 2003;9(2):133-6.

14. Saggi BH, Bloomfield GL, Sugerman HJ, Blocher CR, Hull JP, Marmarou AP, Bullock MR. Treatment of intracranial hypertension using nonsurgical abdominal decompression. J Trauma. 1999;46(4):646-51.

15. Surgeman HJ, Bloomfield GL, Saggi BW. Multisystem organ failure secondary to increased intra-abdominal pressure. Infection. 1999;27(1):61-6.

16. Fritsch DE, Steinmann RA. Managing trauma patients with abdominal compartment syndrome. Crit Care Nurse. 2000;20(6):4858 .
Como citar este artigo:

von Bahten LC, Guimarães PSF. Manuseio da síndrome compartimental abdominal em unidade de tratamento intensivo. Rev Col Bras Cir. [periódico na Internet] 2006 Mai-Jun; 33(3). Disponível em URL: http://www.scielo.br/rcbc

Endereço para correspondência:

Dr. Paulo de Souza Fonseca Guimarães

Rua Heitor Baggio Vidal, 1646

Bairro Alto

82820120 - Curitiba - PR

E-mail: paulo-guimaraes@pop.com.br 